\title{
Efficacy of bevacizumab therapy for unresectable malignant glioma: A retrospective analysis
}

\author{
HAJIME YONEZAWA, HIROFUMI HIRANO, HIROYUKI UCHIDA, MIKA HABU, \\ RYOSUKE HANAYA, TATSUKI OYOSHI, YUKO SADAMURA, TOMOKO HANADA, \\ HIROSHI TOKIMURA, FM MOINUDDIN and KAZUNORI ARITA \\ Department of Neurosurgery, Graduate School of Medical and Dental Sciences, \\ Kagoshima University, Kagoshima 890-8520, Japan
}

Received July 16, 2016; Accepted October 7, 2016

DOI: $10.3892 / \operatorname{mco} .2016 .1086$

\begin{abstract}
Bevacizumab (BEV), an inhibitor of vascular endothelial growth factor $\mathrm{A}$, has been used for primary and recurrent malignant gliomas in Japan since June, 2013. Previous randomized controlled studies demonstrated that $\mathrm{BEV}$ prolonged the progression-free survival, but not the overall survival (OS) of patients with newly diagnosed glioblastoma. The aim of the present study was to elucidate the effect of BEV on the OS of patients with unresectable malignant gliomas. Of the 440 cases of malignant glioma initially treated in our institute between 2000 and 2015, 88 were not suitable for maximal resection due to patient age, physical condition, tumor location and extent, or the patient's wishes. Based on the biopsy results, the pathological diagnosis was glioblastoma, anaplastic astrocytoma and anaplastic oligodendroglioma in 60, 19 and 9 patients, respectively. Kaplan-Meier and log-rank analyses were performed to investigate the effect of BEV on OS. OS was longer in the $\mathrm{BEV}$ group $(n=24)$ compared with that in the non-BEV group [ $\mathrm{n}=64$; median survival time (MST), 566 vs. 243 days, respectively; hazard ratio $(\mathrm{HR})=0.413 ; 95 \%$ confidence interval (CI): $0.216-0.787 ; \mathrm{P}=0.003$ ]. In the 41 patients who received temozolomide (TMZ) and radiotherapy and the 31 patients with glioblastoma who received TMZ and radiotherapy, OS was longer in the BEV group compared with that in the non-BEV group (MST, 568 vs. 334 days, $H R=0.404$, 95\% CI: $0.175-0.933, \mathrm{P}=0.016$; and MST, 566 vs. 160 days, $\mathrm{HR}=0.253$, 95\% CI: 0.099-0.646, $\mathrm{P}=0.001$, respectively). In the Cox hazard model analysis of 41 patients who underwent TMZ-based chemoradiotherapy after biopsy, the use of BEV was the strongest independent beneficial factor associated
\end{abstract}

Correspondence to: Dr Hirofumi Hirano, Department of Neurosurgery, Graduate School of Medical and Dental Sciences, Kagoshima University, 8-35-1 Sakuragaoka, Kagoshima 890-8520, Japan

E-mail: hirahira@m2.kufm.kagoshima-u.ac.jp

Key words: bevacizumab, overall survival, biopsy, malignant glioma, glioblastoma with prolonged $\mathrm{OS}(\mathrm{HR}=0.101 ; \mathrm{P}=0.0002)$. Our retrospective survey suggested that BEV prolongs the OS of patients with unresectable malignant gliomas. However, these results must be verified by a well-designed prospective randomized controlled trial.

\section{Introduction}

Bevacizumab (BEV), an inhibitor of vascular endothelial growth factor A (VEGFA), has been used in Japan as an insurance-covered drug for malignant gliomas since June, 2013. BEV prolongs the overall survival (OS) of patients with various types of cancer in other organs. However, phase III studies on $\mathrm{BEV}$ in combination with chemoradiotherapy demonstrated significant prolongation of the progression-free survival (PFS), but not of the overall survival (OS), in newly diagnosed glioblastomas, apart from a specific genomic subgroup (1-3). These phase III studies included patients undergoing surgical removal of varying extent. The extent of surgical removal of the tumor is well-known to strongly affect OS in glioblastoma (4-6). However, maximal surgical resection may be hindered by various factors, including patient age, physical condition, tumor location and extent, and multiplicity of the lesions. Biopsy via the stereotactic approach or small craniotomy is often selected in these settings. The number of patients with malignant gliomas who undergo biopsy or limited resection may increase due to the aging population. Limited resection was performed in 10-20\% of the cases in a large series of malignant gliomas. The prognosis in these patients was reported to be rather poor $(5,6)$. However, patients who had undergone biopsy or limited resection, referred to as a surgically nearly-naive population, may be an adequate cohort for elucidating the independent effect of BEV on OS, unaffected by the extent of surgical removal. The survival curves of patients with unresectable malignant glioma were retrospectively investigated and compared according to the use of BEV.

\section{Patients and methods}

Patients. Of the 440 patients with malignant glioma who were initially treated in our institute between December, 2000 and January, 2016, 88 (44 men and 44 women) were targeted for biopsy rather than radical surgical resection (Table I). 
The patient age ranged from 5 to 88 years (median, 74 years). The reasons for cases deemed 'unresectable' were patient age $(n=36)$, physical and/or neurological condition $(n=42)$, unfavorable site and extent of the tumor for resective surgery $(n=45)$ and the patient's wishes $(n=1)$, also including patients with multiple reasons. Biopsy was performed via the stereotactic route in 75, small craniotomy in 10 and endoscopic transventricular in 3 patients. The pathological diagnosis was glioblastoma in 60, anaplastic astrocytoma in 19 and anaplastic oligodendroglioma in 9 patients.

\section{Treatments}

Radiotherapy (RT). Extended local RT at 30-60 Gy with 15-30 fractionations was delivered 5 days/week. The clinical target volume was the enhanced area on the T1-weighted image plus a $2-\mathrm{cm}$ margin. In patients with a small residual enhancement lesion observed after $40 \mathrm{~Gy}$ of extended local RT, hypofractionated stereotactic RT using a CyberKnife unit was delivered with 35 Gy/5-8 fractions (Fig. 1).

Primary adjuvant treatment. The time period during which the subjects underwent initial treatment was divided into three periods according to the chemotherapeutic agents used for primary adjuvant treatment as follows: First period, December, 2000-June, 2006; second period, July, 2006-July, 2013; and third period, July, 2013-present. During the first period, procarbazine, nimustin and vincristine (PAV regimen) were administered every 6 weeks as follows: Nimustin $80 \mathrm{mg} / \mathrm{m}^{2}$ intravenously on day 1 ; procarbazine $60 \mathrm{mg} / \mathrm{m}^{2}$ orally daily on days $8-21$; and vincristine $1.4 \mathrm{mg} / \mathrm{m}^{2}$ up to $2 \mathrm{mg}$ intravenously on days 8 and 29. During the second period, oral temozolomide (TMZ) at $75 \mathrm{mg} / \mathrm{m}^{2} /$ day was administered concomitantly with RT. During the third period, oral TMZ at $75 \mathrm{mg} / \mathrm{m}^{2} /$ day and BEV at $10 \mathrm{mg} / \mathrm{kg}$ intravenously every 2 weeks were administered concomitantly with RT.

Maintenance treatment. As maintenance therapy, administration of the PAV regimen was continued in the same manner as the primary PAV therapy during the first period. TMZ was administered at $150-200 \mathrm{mg} / \mathrm{m}^{2}$ for 5 days every 4 weeks during the second and third periods. In the third period, BEV was administered every 2 weeks concomitantly with maintenance TMZ therapy. These therapies were continued until tumor progression leading to deterioration of the patient's condition, rendering the patient unsuitable to undergo maintenance therapy, or until the development of severe adverse effects of grade 3 or 4 according to the Common Terminology Criteria for Adverse Events (https://ctep.cancer.gov/protocolDevelopment/electronic_applications/ctc.htm\#ctc_40).

In total, combined chemoradiotherapy was administered to 64 patients. Of these patients, the initial cytotoxic chemotherapeutic agents used were PAV in 23 and TMZ in 41 patients. $\mathrm{BEV}$ was included in the initial therapy in 19 patients, of whom 17 received BEV combined with TMZ and RT. BEV was administered from the mid-course of the maintenance TMZ therapy in 5 patients. Thus, BEV was administered to 24 of the 88 patients (shaded boxes in Fig. 1).

A total of 16 patients did not receive any adjuvant treatment due to their age and/or general condition, and received supportive care instead; 8 patients received either chemotherapy or RT alone.
Table I. Baseline demographic characteristics of the patients.

\begin{tabular}{lc}
\hline Characteristics & No. \\
\hline Total number & 88 \\
Gender (male:female) & $44: 44$ \\
Age at biopsy (years), mean \pm SD (median) & $67.2 \pm 18.5(74)$ \\
Route of biopsy & \\
Stereotactic biopsy & 75 \\
Small craniotomy & 10 \\
Endoscopic biopsy & 3 \\
PS (ECOG) & \\
0, 1,2 & 43 \\
3,4 & 45 \\
Histopathological diagnosis & \\
WHO grade III & 28 \\
Anaplastic astrocytoma & 19 \\
Anaplastic oligodendroglioma & 9 \\
WHO grade IV glioblastoma & 60 \\
\hline
\end{tabular}

SD, standard deviation; PS, performance status; ECOG, Eastern cooperative oncology group; WHO, world health organization.

Methods. Clinical data were retrieved from the medical records, and information on survival was obtained from the medical records or through telephone interviews with family members. The effect of BEV on OS was investigated using the Kaplan-Meier method and log-rank analysis in three patient groups as follows: In all 88 patients, in the 41 patients who underwent TMZ-based chemoradiotherapy, and in the 31 patients with glioblastoma who underwent TMZ-based chemoradiotherapy. The independent contributing factors to survival were deduced using Cox proportional hazards regression analysis.

Statistical analysis. The Statflex software program, version 6.0 (Artech Co., Osaka, Japan) was used for the statistical analysis of the results. The Kaplan-Meier method and Cox proportional hazard model were used for survival analyses. The difference in survival was assessed with the use of the log-rank test. The Chi-square test was used to evaluate the distribution among the nominal variables. A P-value of $<0.05$ was considered to indicate statistically significant differences.

Ethical considerations. This retrospective study was approved by the Ethics Committee of Kagoshima University Hospital (reference no. 27-160, available at http://com4.kufm. kagoshima-u.ac.jp/information/department/015/015-02.html). The study was conducted in accordance with the Declaration of Helsinki of 1975 as revised in 2000 and the Ethical Guidelines for Medical and Health Research Involving Human Subjects (effective February 9, 2015) by the Ministry of Education, Culture, Sports, Science and Technology, and the Ministry of Health, Labor and Welfare of Japan. To protect the patients' privacy, all data were collected and analyzed under anonymization in an unlinkable manner. 


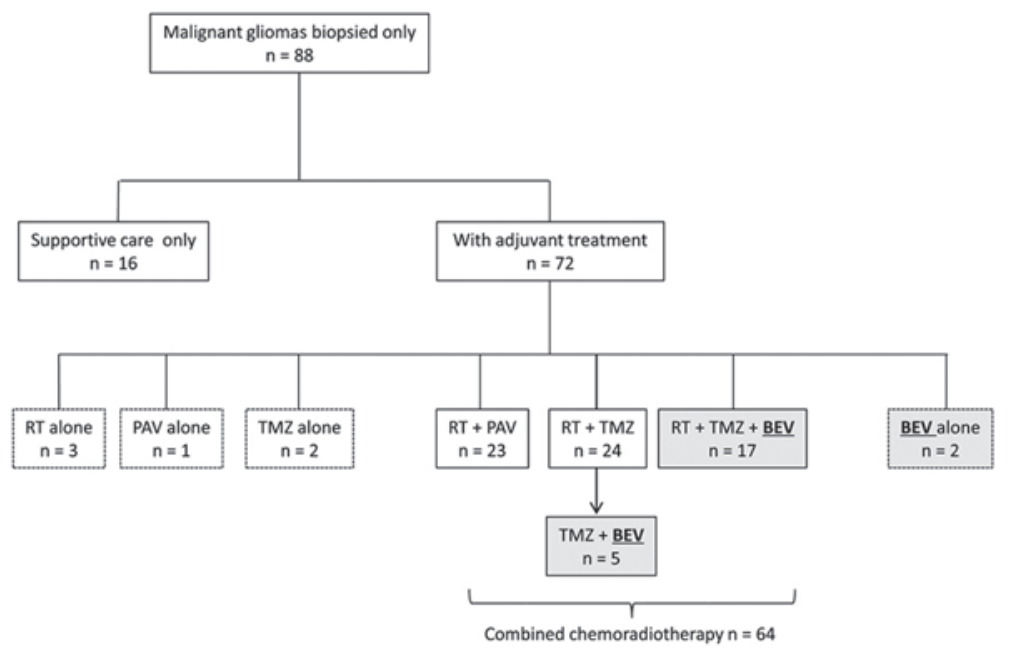

Figure 1. Breakdown of treatments. The shaded boxes indicate patients treated with BEV. RT, radiotherapy; PAV, procarbazine, nimustin and vincristine; TMZ, temozolomide; BEV, bevacizumab.

\section{Results}

Survival analysis. The observation period was 10-2,417 days (median, 263 days). The median survival time (MST) of the 88 patients was 317 days. The Kaplan-Meier analysis and log-rank test results revealed a significantly longer OS in patients with grade III compared with grade IV gliomas ( $\mathrm{P}=0.032$; MST, 431 vs. 282 days, respectively).

The effect of BEV was analyzed in the three patient groups. The clinical characteristics of the patients who received BEV and those who did not in the three patient groups are summarized in Table II. In all 88 patients and in the 41 patients with malignant glioma who received TMZ-based chemoradiotherapy, the non-BEV group included more patients with grade III tumors compared with the BEV group. The differences were statistically significant $(\mathrm{P}=0.004$ and 0.014 , respectively, Chi-square test; Table IIA and B). Among the 88 patients, those who received BEV more frequently underwent cytotoxic chemotherapy and RT, including the use of the CyberKnife (Table IIA).

Of the 88 patients, OS was longer in the 24 patients treated with BEV compared with that in the 64 patients who did not receive BEV [MST, 566 vs. 243 days; hazard ratio $(\mathrm{HR})=0.413$; 95\% confidence interval (CI): 0.216-0.787; P=0.003; Fig. 2]. In the 41 patients who received TMZ-based chemoradiotherapy, OS was longer in the 22 patients treated with BEV compared with that in the 19 patients not treated with BEV (MST, 568 vs. 334 days, respectively; HR=0.404; 95\% CI: 0.175-0.933; $\mathrm{P}=0.016$; Fig. 3). In the 31 patients with glioblastomas who received TMZ-based chemoradiotherapy, OS was longer in the 20 patients treated with BEV compared with that in the 11 patients not treated with BEV (MST, 566 vs. 160 days, respectively; HR=0.253; 95\% CI: 0.099-0.646; P=0.001; Fig. 4). The independent contributing factors for OS were deduced using Cox proportional hazards regression models. In 72 patients who underwent any type of adjuvant therapy, Cox proportional hazard analysis using the World Health Organization (WHO) grade, age, Eastern Cooperative Oncology Group (ECOG) performance scale score after biopsy, radiation, CyberKnife, nimustin, TMZ and BEV as covariates, revealed that WHO

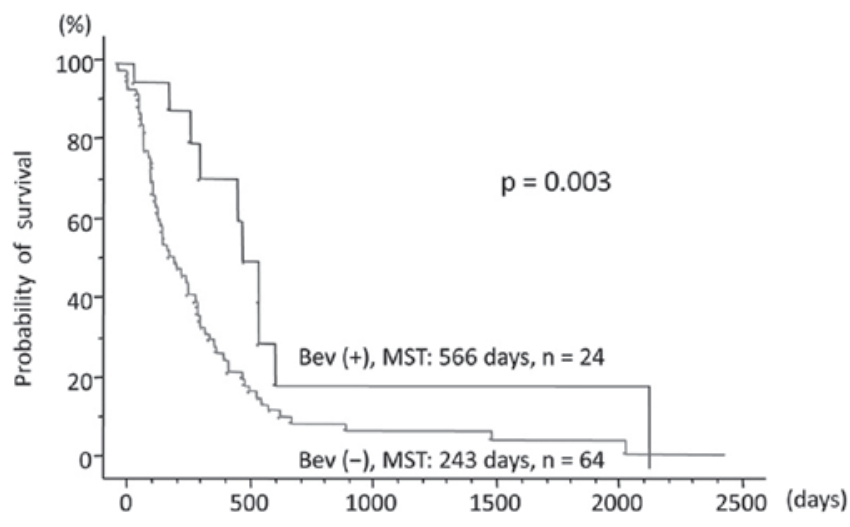

Figure 2. Analysis of overall survival in 88 patients treated with and without bevacizumab (BEV). The overall survival of the patients treated with BEV was significantly better compared with that of patients not treated with BEV $(\mathrm{P}=0.018, \log$-rank test). MST, median survival time.

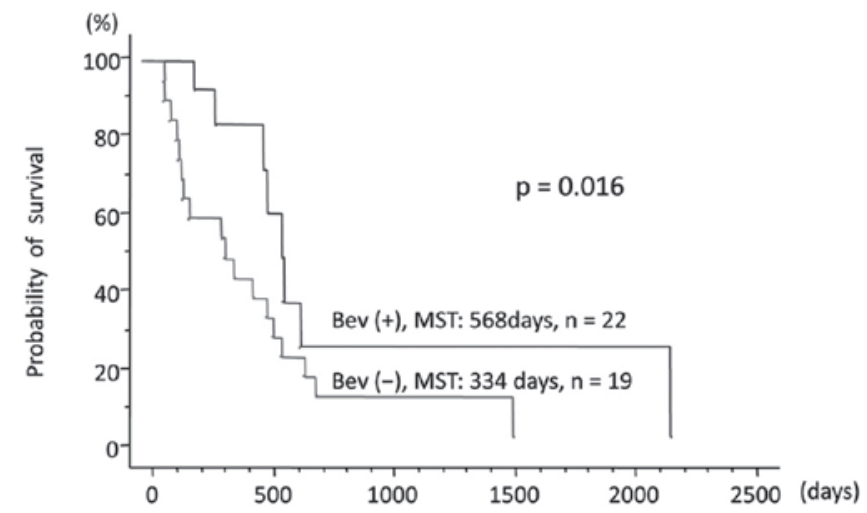

Figure 3. Analysis of the effect of bevacizumab (BEV) on overall survival in the 41 patients who underwent temozolomide-based chemoradiotherapy after biopsy. The overall survival of the patients treated with BEV was significantly better compared with that of patients not treated with $\mathrm{BEV}(\mathrm{P}=0.043$, log-rank test). MST, median survival time.

grade III, good PS (0-2) and use of BEV were independent prognostic factors of survival $(\mathrm{HR}<1 ; \mathrm{P}<0.05$; Table III). In the 
Table II. Clinical factors of the three patient groups.

A, A total of 88 patients with unresectable malignant gliomas

\begin{tabular}{lccc}
\hline Characteristics & BEV & non-BEV & P-value \\
\hline Gender (male:female) & $10: 14$ & $34: 30$ & 0.338 \\
Age at biopsy (years), mean \pm SD & $65.3 \pm 21.2$ & $67.9 \pm 17.5$ & 0.282 \\
PS (0-2:3,4) & $13: 11$ & $30: 34$ & $26: 38$ \\
WHO grade (III:IV) & $2: 22$ & 27 & $\mathbf{0 . 0 0 4}$ \\
Location & & 14 & 0.427 \\
Dominant side & 14 & 18 \\
Non-dominant side & 5 & 5 & \\
Bilateral & 3 & $46: 18$ \\
Posterior fossa & 2 & $45: 19$ \\
Cytotoxic chemotherapy (yes:no) & $22: 2$ & $10: 54$ \\
Radiotherapy (yes:no) & $22: 2$ & $\mathbf{0 . 0 4 8}$ \\
CyberKnife (yes:no) & $10: 14$ & $\mathbf{0 . 0 3 6}$ \\
\end{tabular}

B, A total of 41 patients with unresectable malignant gliomas who received TMZ-based chemoradiotherapy

\begin{tabular}{lccc}
\hline Characteristics & BEV & non-BEV & P-value \\
\hline Gender (male:female) & $9: 13$ & $10: 9$ & 0.453 \\
Age at biopsy (years), mean \pm SD & $64.9 \pm 21.8$ & $69.5 \pm 18.3$ & 0.236 \\
PS $(02: 3,4)$ & $13: 9$ & $10: 9$ & 0.678 \\
WHO grade (III:IV) & $2: 20$ & $8: 11$ & $\mathbf{0 . 0 1 4}$ \\
Location & & 9 & 0.787 \\
Dominant side & 12 & 3 & \\
Non-dominant side & 5 & 3 & $4: 15$ \\
Bilateral & 3 & 2 & \\
Posterior fossa & $10: 12$ & & 0.189 \\
CyberKnife (yes:no) & & \\
\hline
\end{tabular}

C, A total of 31 patients with unresectable glioblastomas who received TMZ-based chemoradiotherapy

\begin{tabular}{lccc}
\hline Characteristics & BEV & non-BEV & P-value \\
\hline Gender (male:female) & $8: 12$ & $4: 7$ & 0.842 \\
Age at biopsy (year), mean \pm SD & $67.9 \pm 20.4$ & $67.2 \pm 21.9$ & $3: 8$ \\
PS (02:3,4) & $12: 8$ & & 0.463 \\
Location & & 5 & 0.554 \\
Dominant side & 11 & 4 & 1 \\
Non-dominant side & 4 & $1: 10$ \\
Bilateral & 3 & 2 & \\
Posterior fossa & $8: 12$ & 0.106 \\
CyberKnife (yes:no) & 2 & & \\
\hline
\end{tabular}

Statistical analysis was performed using the unpaired $t$-test for age at biopsy and the $2 \mathrm{x} 2 \mathrm{Chi}$-square test for other characteristics. Bold print indicates statistically significant results $(\mathrm{P}<0.05)$. BEV, bevacizumab; SD, standard deviation; PS, performance status; WHO, world health organization; TMZ, temozolomide.

41 patients who underwent TMZ-based chemoradiotherapy, Cox proportional hazard analysis using WHO grade, age, ECOG performance scale score after biopsy, CyberKnife and use of BEV as covariates, revealed that WHO grade III, good PS (0-2) and use of BEV were also independent prognostic factors of survival $(\mathrm{HR}<1 ; \mathrm{P}<0.05$; Table IV). The use of $\mathrm{BEV}$ 
Table III. Cox proportional hazard model analysis of the 72 patients who underwent adjuvant therapy after biopsy.

\begin{tabular}{lcrc}
\hline Characteristics & $\begin{array}{c}\text { Degree of } \\
\text { freedom }\end{array}$ & P-value & $\begin{array}{c}\text { Hazard } \\
\text { ratio }\end{array}$ \\
\hline WHO grade III & 1 & $\mathbf{0 . 0 1 1 0}$ & 0.453 \\
Age & 1 & 0.9662 & 1.000 \\
PS $(0,1,2)$ & 1 & $\mathbf{< . 0 0 0 1}$ & 0.201 \\
Radiotherapy & 1 & 0.2538 & 0.537 \\
CyberKnife & 1 & 0.2596 & 1.510 \\
Nimustin & 1 & 0.1092 & 0.449 \\
TMZ & 1 & 0.2021 & 0.545 \\
Bevacizumab & 1 & $\mathbf{0 . 0 0 0 2}$ & 0.199 \\
\hline
\end{tabular}

Bold print indicates statistically significant results $(\mathrm{P}<0.05)$. PS, performance status; WHO, world health organization; TMZ, temozolomide.

Table IV. Cox proportional hazard model analysis of the 41 patients who underwent TMZ-based chemoradiotherapy after biopsy.

\begin{tabular}{lccc}
\hline Characteristics & $\begin{array}{c}\text { Degree of } \\
\text { freedom }\end{array}$ & P-value & $\begin{array}{c}\text { Hazard } \\
\text { ratio }\end{array}$ \\
\hline WHO grade III & 1 & $\mathbf{0 . 0 0 3 8}$ & 0.207 \\
Age & 1 & 0.2811 & 1.013 \\
PS $(0,1,2)$ & 1 & $\mathbf{0 . 0 0 1 7}$ & 0.135 \\
CyberKnife & 1 & 0.1518 & 2.242 \\
Bevacizumab & 1 & $\mathbf{0 . 0 0 0 2}$ & 0.101 \\
\hline
\end{tabular}

Bold print indicates statistically significant results $(\mathrm{P}<0.05)$. PS, performance status; WHO, world health organization; TMZ, temozolomide.

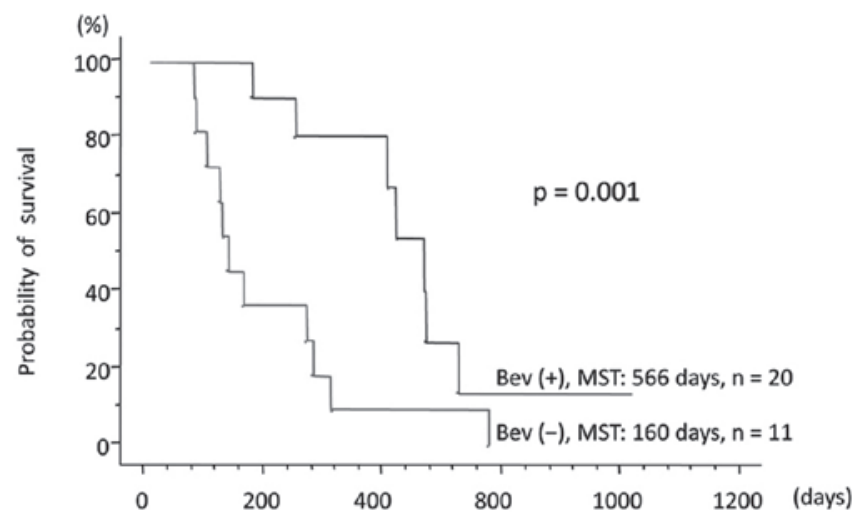

Figure 4. Analysis of the effect of bevacizumab (BEV) on overall survival in 31 patients with glioblastoma who underwent temozolomide-based chemoradiotherapy after biopsy. The overall survival of the patients treated with BEV was significantly better compared with that of patients not treated with BEV $(\mathrm{P}=0.048, \log$-rank test). MST, median survival time.

was the strongest favourable factor $(\mathrm{HR}=0.101 ; \mathrm{P}=0.0002)$ among the three factors. No BEV-specific adverse events, such as wound dehiscence, intracranial hemorrhage, or extracranial hemorrhage, were reported.

\section{Discussion}

The results of this retrospective analysis using Kaplan-Meier and Cox hazard model analyses suggest a strong beneficial effect of BEV on the OS of patients with malignant glioma. Among all 88 patients and the 41 patients who underwent TMZ-based chemoradiotherapy, the proportion of patients with grade III tumors was higher among the non-BEV patients; however, this does not invalidate the results showing an effect of $\mathrm{BEV}$ on OS, as WHO grade III was an independent prognostic factor for OS according to the results of the Cox proportional hazards model analysis. The 16 patients who did not receive any adjuvant therapy were included in the total 88 patients; thus, the frequencies of cytotoxic chemotherapy and RT were not balanced between the BEV and non-BEV patients. If the 16 patients were omitted, no significant difference in treatment frequency would have been found between the BEV and non-BEV groups. In addition, these treatments were not independent prognostic factors for OS (Tables III and IV).

$\mathrm{BEV}$ is an anti-VEGFA molecule that strongly inhibits the action of the angiogenic factors spontaneously produced by tumor cells and/or tumor-associated stroma in malignant tumors (7). Through its inhibitory effect, BEV reduced the peritumoral edema in human malignant brain tumors, including glioblastomas and metastatic lesions. BEV also suppressed glioblastoma growth in an in vivo xenograft model $(8,9)$.

$\mathrm{BEV}$ is well-known to significantly prolong PFS in a number of cancer types. However, in terms of OS, it showed some benefit in certain types of primary and metastatic cancers, but not in breast, pancreatic, gastric, or ovarian cancer (10-13). The difference in the benefit of BEV regarding OS among various types of malignant tumors has been attributed to the variation and effects of cytotoxic chemotherapeutic agents concomitantly used with BEV on neoplasms $(12,13)$. Rapid changes in the biological nature of cancers induced by anti-VEGF therapy and/or the cessation of BEV treatments defined by treatment protocols may induce rebound effects on angiogenesis and tumor growth. These effects may eventually curtail the BEV benefit for OS $(10,12)$. Differences in disease-specific OS and length of survival after progression between malignancies may play a role in this difference $(11,12,14,15)$. Various additional lines of treatment after progression, including crossover-allowed design, may also obscure the survival-improving benefit of anti-VEGF therapy $(10,13)$.

Two precedent randomized controlled trials (AVAglio and RTOG-0825) reported longer median PFS with BEV compared with that with placebo, but did not observe a prolongation of OS with the addition of BEV to RT plus TMZ $(1,2)$. One of the suggested reasons for failure to exert an effect on OS is the crossover design of the studies on glioblastoma $(1,2,16)$. Another suspected reason for the absence of a prolonging effect on OS was omission of patients with a Karnofsky performance status of $<70$ in the study cohort $(1,2)$. Omission of such patients may cancel the potential OS-prolonging effect that may be gained through improvement of performance by BEV administration (16). 
The advantage of our study is that the subjects were limited to those who underwent biopsy alone. Between 2000 and 2015, which is the period during which our patients underwent surgery, the 5-aminolevulinic acid-induced fluorescence technique and intraoperative magnetic resonance imaging to identify residual tumor were introduced in our hospital. Thus, the resection rate improved over time (5). This made it difficult to compare the OS of all the patients with malignant glioma in the BEV era (June, 2013-present) with that of those in the pre-BEV era, as the resection rates differed significantly.

In our study design, the resection rate was universally $<10 \%$ and would not have affected the therapeutic results. Therefore, we hypothesized that the independent effect of BEV on malignant gliomas could be extracted through OS comparison between patients who received BEV and those who did not, both groups being almost 'surgically naïve'. Biopsy-only cases were omitted from the RTOG-0825 study, which comprised only $11.3 \%$ of the AVAglio study cohort $(1,2)$.

Although our study suggested a prolonging effect on OS in unresectable malignant glioma cases, a recent report of a phase II trial on unresectable glioblastomas (TEMAVIR study) did not demonstrate prolongation of OS with BEV (17). It should be noted that the starting ratio of radiation was $66.7 \%$ in the experimental arms and $97.6 \%$ in the control arms in the latter studies. As regards adjuvant therapy, BEV/irinotecan without TMZ was allocated to the experimental arm; not only TMZ, but also salvage BEV was permitted in the control arm. The inequality in the use of concomitant chemotherapeutic agents, the infeasibility of treatment protocols for the experimental arm, and the use of salvage BEV may have made the benefit of BEV on OS difficult to identify. In addition, these adjuvant treatments were continued for only 6 months or discontinued upon disease progression. Our policy to continue adjuvant treatment, including $\mathrm{BEV}$, after progression was identified may have contributed to the prolongation of OS in the BEV treatment groups. In fact, BEV was continued after progression was diagnosed in 8 patients of our series.

Among the 27 patients who received initial radiation + TMZ treatment in our study, $90.9 \%$ of patients in the group that received additional $\mathrm{BEV}$ also received $\mathrm{TMZ}$ maintenance therapy; this percentage was only $43.8 \%$ in the group without additional BEV. Adding BEV to the initial chemoradiotherapy may protect the general and neurological status of our senior population, which would eventually facilitate maintenance chemotherapy and contribute to the OS benefit.

This study has several disadvantages inherent to the nature of small retrospective cohorts as follows: Lack of i) randomization, ii) consistency of concomitant chemotherapeutic agents, iii) dose and timing of BEV administration, and iv) stratification according to isocitrate dehydrogenase-1 gene status and gene profiling (3). A future study protocol is currently being prepared with consideration of the aforementioned factors.

This retrospective survey of 88 unresectable malignant gliomas demonstrated the potential of BEV to prolong the OS of patients with disadvantageous conditions. Considering the rapidly aging population, the beneficial effect of BEV on OS should be verified by prospective studies, particularly in subsets of patients who are elderly and/or in poor general condition.

\section{Acknowledgements}

We would like to thank Editage (www.editage.jp) for the English language editing. This study was partially aided by research grants, Kiban Research Grant C (15K10338 to H.H.) from the Ministry of Education, Culture, Sports, Science and Technology of Japan.

\section{References}

1. Chinot OL, Wick W, Mason W, Henriksson R, Saran F, Nishikawa R, Carpentier AF, Hoang-Xuan K, Kavan P, Cernea D, et al: Bevacizumab plus radiotherapy-temozolomide for newly diagnosed glioblastoma. N Engl J Med 370: 709-722, 2014.

2. Gilbert MR, Dignam JJ, Armstrong TS, Wefel JS, Blumenthal DT, Vogelbaum MA, Colman H, Chakravarti A, Pugh S, Won M, et al: A randomized trial of bevacizumab for newly diagnosed glioblastoma. N Engl J Med 370: 699-708, 2014.

3. Sandmann T, Bourgon R, Garcia J, Li C, Cloughesy T, Chinot OL, Wick W, Nishikawa R, Mason W, Henriksson R, et al: Patients with proneural glioblastoma may derive overall survival benefit from the addition of bevacizumab to first-line radiotherapy and temozolomide: Retrospective analysis of the AVAglio trial. J Clin Oncol 33: 2735-2744, 2015.

4. Sanai N, Polley MY, McDermott MW, Parsa AT and Berger MS: An extent of resection threshold for newly diagnosed glioblastomas. J Neurosurg 115: 3-8, 2011

5. Kawano H, Hirano H, Yonezawa H, Yunoue S, Yatsushiro K, Ogita M, Hiraki Y,Uchida H, Habu M, Fujio S, et al: Improvement in treatment results of glioblastoma over the last three decades and beneficial factors. Br J Neurosurg 29: 206-212, 2015.

6. Committee of Brain Tumor Registry of Japan: Report of Brain Tumor Registry of Japan (2001-2004). 13th ed. Neurol Med Chir (Tokyo) 54 (Suppl): 1-102, 2014.

7. Ferrara N, Hillan KJ and Novotny W: Bevacizumab (Avastin), a humanized anti-VEGF monoclonal antibody for cancer therapy. Biochem Biophys Res Commun 333: 328-335, 2005.

8. Kim KJ, Li B, Winer J, Armanini M, Gillett N, Phillips HS and Ferrara N: Inhibition of vascular endothelial growth factor-induced angiogenesis suppresses tumour growth in vivo. Nature 362: 841-844, 1993.

9. Mathieu V, De Nève N, Le Mercier M, Dewelle J, Gaussin JF, Dehoux M, Kiss R and Lefranc F: Combining bevacizumab with temozolomide increases the antitumor efficacy of temozolomide in a human glioblastoma orthotopic xenograft model. Neoplasia 10: 1383-1392, 2008.

10. Ebos JM and Kerbel RS: Antiangiogenic therapy: Impact on invasion, disease progression and metastasis. Nat Rev Clin Oncol 8: 210-221, 2011.

11. Ocaña A, Amir E, Vera F, Eisenhauer EA and Tannock IF: Addition of bevacizumab to chemotherapy for treatment of solid tumors: Similar results but different conclusions. J Clin Oncol 29: 254-256, 2011.

12. Amit L, Ben-Aharon I, Vidal L, Leibovici L and Stemmer S: The impact of bevacizumab (avastin) on survival in metastatic solid tumors-a meta-analysis and systematic review. PLoS One 8: e51780, 2013.

13. Li Q, Yan H, Zhao P, Yang Y and Cao B: Efficacy and safety of bevacizumab combined with chemotherapy for managing metastatic breast cancer: A meta-analysis of randomized controlled trials. Sci Rep 5: 15746, 2015.

14. Broglio KR and Berry DA: Detecting an overall survival benefit that is derived from progression-free survival. J Natl Cancer Inst 101: 1642-1649, 2009.

15. Morita S, Sakamaki K and Yin G: Detecting overall survival benefit derived from survival postprogression rather than progression-free survival. J Natl Cancer Inst 107: djv133, 2015.

16. Narita Y: Bevacizumab for glioblastoma. Ther Clin Risk Manag 11: 1759-1765, 2015.

17. Chauffer B, Feuvret L, Bonnetain F, Taillandier L, Frappaz D, Taillia H, Schott R, Honnorat J, Fabbro M, Tennevet I, et al: Randomized phase II trial of irinotecan and bevacizumab as neo-adjuvant and adjuvant to temozolomide-based chemoradiation compared with temozolomide-chemoradiation for unresectable glioblastoma: Final results of the TEMAVIR study from ANOCEF. Ann Oncol 25: 1442-1447, 2014. 